\title{
Literatur
}

1. Stani, E., Pharmazie 11, 633 (1956). - 2. Stark, E., Chemiker Ztg. 82, 323 (1958). - 3. Start, E., Arch. Pharmazie Berlin 292, 411 (1959). - 4. Stafi, E., Arch. Pharmazie, Berlin 293, 531 (1960). - 5. Folch, J., M. Lees und G. H. Sloans Stanley, J. Lipid Res. 1, 391 (1959). - 6. Folch, J., I. Ascoli, M. LeEs, J. A. Meath und P. N. Le Baron, J. biol. Chemistry 191, 833 (1951). - 7. BöHLE, E. und E. StarCK, Untersuchungen über die fäkale Lipidexkretion beim Menschen S. 52. Pallas Verlag München (1966). - 8. Serfer, A., Fette, Seifen einschl. Anstrichmittel 61, 345 (1959). - 9. Blank, M. I., J. A. Schmit und O. S. Privett, J. Amer. Oil Chemists' Soc. 41, 371 (1964). - 10. PrÉ, A. und A. Giner, Nature London 212, 402 (1966). - 11. Privett, O. S., M. L. Blank, D. W. Colding und E. C. Nickell, J. Amer. Oil Chemists' Soc. 42, 381 (1965). - 12. Privetr, O. S., M. L. Blank und W. LundaerG, J. Amer. Oil Chemists' Soc. 38, 297 (1961). 13. Fukuda, J., E. Mirukami und K. Imaichr, J. Biochemistry (Tokyo) 61, 657 (1967). - 14. Mangold, H. K., J. Amer. Oil Chemists' Soc. 41, 762 (1964). - 15. Mangold, H. K., J. Amer. Oil Chemists' Soc. 38, 708 (1961). - 16. Vroque, E. und L. J. Morris, J. Amer. Oil Chemists' Soc. 38, 482 (1961). - 17. VIOQUE, E., L. J. Morris und R. T. Holman, J. Amer. Oil Chemists' Soc. 38, 489 (1961): - 18. Williams, J. A., A. Sharma, L. S.
Morris und R. Holman, Proc. Soc. exp. Biol. Med. 105, 192 (1960). - 19. Gänshirt, H., F. W. Koss und W. K. Morianz, Arzneimittelforsch. Aulendorf 10, 943 (1960).-20. SNYDER, F.,Analytic. Biochem. 9, 183 (1964). - 21. SNYDER, F. und N. STEPHENS, Analytic. Biochem. 4, 128 (1962). - 22. SNYDER, F. und N. Stephens, Biochim. biophysica Acta, Amsterdam 34, 244 (1959). 23. Purdy, S. J. und E. V. Truter, Chem. and Ind. (1962), 506. - 24. Purdy, S. J. und E. V. Truter, Analyst 87, 802 (1962). - 25. SEHER, A., Mikrochim. Acta 1961, 308. - 26. Serer, A., Nahrung 4, 466 (1960). - 27. Lines, J. G., in: Biochemical problems of lipids S. 17. Hrsg. A. C. Frazer, Elsevier Publ. Co., New York (1963). - 28. Sawickr, E., T. W. Stanley und H. Thomson, Microchem. J. 8,257 (1964). - 29. BeCK, K., R. Sickinger und R. GNANK, Med. Klin. 59, 1243 (1964). - 30. Drube, H. Chr. und H. Bütrner, Klin. Wschr. 41, 749 (1963). 31. Kamer, van de, J. H., H. A. ten Bokkel Huinink und $\mathrm{H}$. A. Weyers, J. biol. Chemistry 177, 347 (1949). - 32. Krnsell, L. W., R. W. Friskey, G. D. Michaels und S. Splitter, Lancet London 1958/I 334. - 33. Mietrinen, T. A., E. H. Ahrens und S. W. GRUNDY, J. Lipid Res. 6, 411. (1965) - 34. Moore, R. B., J. T. Anderson, A. Keys und J. D. Frantz, J. Laborat Clin. Med. S. Louis 60, 1000 (1962).
Priv.-Doz. Dr. E. Böhle 6000 Frankfurt/Main 70 Ludwig-Rehn-Str. 14

\section{Analyse der Erythrocytenporphyrine}

\author{
Von M. Doss und U. BoDE \\ Aus dem Hygiene-Institut und Medizinaluntersucbungsamt der Universität Marburg (Labn) \\ (Direktor: Prof. Dr. R. Siegert) \\ (Eingegangen am 18. März 1968)
}

Die Porphyrine von Erythrocyten des Menschen wurden nach dünnschichtchromatographischer Trennung spektrophotometrisch als Methylester analysiert.

Untersuchungen von 59 Personen ergaben einen Normalbereich des Erythrocytenprotoporphyrins von 5 bis $80 \mu \mathrm{g} / 100 \mathrm{~m} l$ Erythrocyten. Die Protoporphyrinwerte des vorliegenden Probekollektivs zeigen eine log-normale Verteilung.

\section{The analysis of the porphyrins of erytbrocytes}

The porphyrins of human erythrocytes were separated by thin layer chromatography and determined spectrophotometrically as their methyl esters.

From a study of 59 persons, the normal range of erythrocyte protoporphyrin was $5-80 \mu \mathrm{g} / \mathrm{m} l$ of erythrocytes. The protoporphyrin values of the collective samples showed a log-normal distribution.

Die Methodik, freie Porphyrine im biologischen Material in Methylester zu überführen und dưrch Dünnschichtchromatographie ạn Kieselgel $\mathrm{H}(1,2) \mathrm{zu}$. isolieren, wurde zur quantitativen Analyse der Porphyrine von Erythrocyten des Menschen angewandt.

\section{Material und Methoden}

Die Erythrocyten aus 10 oder $20 \mathrm{~m} l$ Venenblut (Abnahme mit $0,1[0,2] \mathrm{m} l$ Liquemin) hämatologisch gesunder Personen werden mit physiol. $\mathrm{NaCl}-\mathrm{Lösung}$ gewaschen, gefriergetrocknet und mit Methanol- $\mathrm{H}_{2} \mathrm{SO}_{4}$ 95:5(v/v) versetzt. Nach 12 bis $14 \mathrm{Stdn}$. bei Raumtemperatur haben sich die Tetrapyrrolmethylester gebildet, die nach Abtrennung der corpuskulären Teile (Zentrifugation bei $25000 \mathrm{~g}, 10 \mathrm{Min}$.) und Zugabe von Wasser mit Chloroform ausgeschüttelt werden. Den neutralisierten, gewaschenen und auf etwa $2 \mathrm{~m} /$ eingeengten Gesamtextrakt trägt man auf eine KieselgelH-Platte (Breite $\times$ Höhe: $40 \times 20 \mathrm{~cm}$; Schichtdicke $2 \mathrm{~mm}$ ) streifenförmig auf und entwickelt das Chromatogramm im Lösungsmittelsystem Benzol/Essigsäureäthylester/Methanol 85:13,3: $1,7(\nabla / v)$ zweimal bis zu einer Höhe von $13(15) \mathrm{cm}$. Die unter dem UV-Licht $(355 \mathrm{~nm})$ rotfluoreszierenden Zonen der Trägerschicht werden in ein Glasgerät mit eingelassener Fritte (3) gesaugt, aus dem man Protoporphyrin- und Koproporphyrinmethylester mit Chloroform eluiert. Die spektrophotometrische Messung der Porphyrine erfolgt am jeweiligen Absorptionsmaximum (SoretBande). Für die Quantifizierung der Methylester stehen millimolare Extinktionskoeffizienten zur Verfügung (4). Der Porphyringehalt der Erythrocyten wurde nach folgender Formel berechnet: 
$\mu \mathrm{g}$ Proto (Kopro)porphyrin $=\frac{\mathrm{E} \cdot \mathrm{Mol} . \mathrm{Gew} \cdot \mathrm{V}_{1} \cdot 10^{4}}{\epsilon_{\mathrm{mMI}} \cdot \mathrm{d} \cdot \mathrm{V}_{2} \cdot \mathrm{Hct} \%}$

$\mathrm{V}_{1}=$ Meßvolumen $\mathrm{CHCl}_{3}$

$\mathrm{V}_{2}=$ aufgearbeitetes Blutvolumen

$\mathrm{Hct}=$ Hämatokrit

Die Ausbeute des Verfahrens lag nach Wiederfindungsversuchen mit Protoporphyrin-IX-dimethylester zwischen 70 und 75\%. Der Variationskoeffizient (Standardabweichung in Prozent des arithmetischen Mittels) als weiteres Maß für die Richtigkeit der Methode betrug $7,5 \%(n=6)$.

\section{Ergebnisse und Diskussion}

Porphyrine, Protohämin und Lipoide im Chloroformextrakt

Bei der Veresterung der Porphyrine und des Protohämins mit Methanol-Schwefelsäure entstehen aus den Glycerophosphatiden der Erythrocyten (5) Fettsäuremethylester (6), die im Dünnschichtchromatogramm über $\operatorname{dem} R_{\mathrm{F}}$-Wert des Protoporphyrin-dimethylesters nahe der Lösungsmittelfront liegen und sich durch eine hellblaue Fluoreszenz unter dem UV-Licht $(355 \mathrm{~nm})$ auszeichnen. Die Zusammensetzung dieses Fettsäuremethylestergemisches entspricht nach der gaschromatographischen Analyse weitgehend dem Fettsäuremethylestergemisch, das durch alkali-katalysierte Umesterung $(7,8)$ von Glycerophosphatiden der Erythrocyten dargestellt wurde (Glycerophosphatide wurden aus einem Erythrocytenlipoidgesamtextrakt durch Chromatographie an Kieselsäure (Mallinckrodt) und Hyflo Super Celite $(2: 1, w / w)$ gewonnen (9). Beimengungen von Fettsäuremethylestern zu Porphyrinmethylestern beeinträchtigen die spektrophotometrische Analyse in Chloroform gelöster Porphyrinmethylester nicht $\left.(6)^{1}\right)$. Außer den Fettsäuremethylestern befinden sich Cholesterin und Glycerophosphatide neben großen Mengen Protohämin-dimethylester im Chloroformextrakt der Porphyrinmethylester. Der Protoporphyrinmethylester trennt sich von diesen Substanzen im Dünnschichtchromatogramm mit einem wesentlich höheren $R_{\mathrm{F}^{-}}$-Wert ab.

Säulenchromatographische Trennungen von Porphyrinmethylestern (10) mit verschiedenen Adsorbentien, einschließlich präparierter Kieselsäure Mallinckrodt (11), ließen sich mit verschiedenen Lösungsmittelsystemen erzielen, waren aber speziell für die Erythrocytenporphyrinisolierung infolge des Häminüberschusses im Chloroformextrakt (Eisenprotoporphyrin des Hämoglobins / freies Erythrocytenprotoporphyrin $\approx 30000: 1$ (12)) nur in Verbindung mit anschließender dünnschichtchromatographischer Reinigung des Protoporphyrins (auf $0,5 \mathrm{~mm}$ dick beschichteten Platten) geeignet.

\section{Normalwerte der Erythrocytenporphyrine}

Die Ergebnisse der an Erythrocyten von 59 Personen durchgeführten Analysen sind in Tabelle 1 zusammen-

1) Die Firma Beckman-Instruments, Technisches Büro Frankfurt a. M., stellte freundlicherweise ein Spektralphotometer DB-G zur Verfügung.
Tab. 1

Isolierung und Bestimmung der Erythrocytenporphyrine als Methylester nach präparativer dünnschichtchromatographischer Auftrennung aus je $20 \mathrm{ml}$ Venenblut*. Angaben in $\mu \mathrm{g} / 100 \mathrm{ml}$ Erythrocyten

\begin{tabular}{|c|c|c|c|c|}
\hline \multirow[t]{2}{*}{ Analysen } & \multicolumn{3}{|c|}{ Protoporphyrin } & \multirow{2}{*}{$\begin{array}{c}\text { Kopropor- } \\
\text { phyrin } \\
\text { Bereich }\end{array}$} \\
\hline & Mittelwert & Bereich & $\log \bar{x} \pm 2 s$ & \\
\hline $\begin{array}{ll}\text { Kollektiv } & 59 \\
\text { Männer } & 31 \\
\text { Frauen } & 28\end{array}$ & $\begin{array}{l}24,92 \\
28,21 \\
21,27\end{array}$ & $\begin{array}{l}6,2-79,0 \\
6,2-79,0 \\
6,8-65,4\end{array}$ & $\begin{array}{l}20,39 \pm 3,77 \\
22,85 \pm 3,94 \\
17,97 \pm 3,52\end{array}$ & $0-1$ \\
\hline
\end{tabular}

* Hämoglobin, Hämatokrit (nach VAN ALLEN), Retikulocyten (4 bis $13 \%$ ) im Normbereich (20).

gefaßt und werden den bei der Extraktion der freien Säuren $(13,14,15)$ ermittelten Werten gegenübergestellt (Táb. 2). Direkte Vergleiche der Ergebnisse verschiedener Untersucher sind nicht möglich, ohne die einzelnen methodischen Variationen zu berücksichtigen. Der mit unserer Methode gefundene Normalbereich korreliert mit den Werten anderer Untersucher. Koproporphyrin ließ sich nur bei 8 der 59 Proben in meßbarer Menge feststellen. Die Werte lagen unter und um $1 \mu \mathrm{g} / 100 \mathrm{~m} l$ Erythrocyten. Über die Beziehung der Erythrocytenporphyrine zur Reticulocytenzahl liegen unterschiedliche Ergebnisse vor (12, 15, 16, 17, 18). Mehrere Untersucher $(12,15,17,18)$ konnten eine lineare Beziehung $z$ wischen dem Protoporphyringehalt der Erythrocyten und der Reticulocytenzahl nicht feststellen. Dagegen besteht eine enge Parallelität $z$ wischen der Erythrocytenkoproporphyrinkonzentration und Reticulocytenzahl bei erhöbter Erythropoese (15, 16). Innerhalb des Normalbereiches läßt. sich jedoch nach einer methodologischen Studie der Analyse von freien Porphyrinen (12) und nach eigenen Untersuchungen eine strenge Wechselbeziehung nicht erkennen. Meßtechnische Faktoren spielen bei der Analyse kleiner Koproporphyrinmengen ( $<1 \mu \mathrm{g} / 100 \mathrm{~m} l$ Erythrocyten) aus relativ großen Blutvolumina eine Rolle. Sämtliche bis heute zur Verfügung stehenden Verfahren der quantitativen Erythrocytenporphyrinbestimmungen sind für ein Routinelaboratorium meistens zu aufwendig. Die Protoporphyrinbestimmung aus Erythrocyten ist entscheidend für die Diagnose der erythropoetischen Protoporphyrie. Diese Diagnose kann mit der dünnschichtchromatographischen FluoreszenzScreening-Methode gestellt werden (19).

Stichprobenverteilung des Erythrocyten-Protoporphyrins und Verteilungsfunktion der Grundgesamtheit

Die Normalwerte wurden zur graphischen (20) und statistischen Ermittlung des Verteilungstyps in acht (neun) Klassen eingeteilt. Abbildung 1 zeigt das Verhalten der Analysenwerte in linearen und logarithmischen Skalen. Das graphische Bild spricht für einen log-normalen Verteilungstyp, der mit der Chi-QuadratMethode nach Pearson (cf. 21) überprüft wird.

Mit dem Chi-Quadrat-Test wird die Hypothese, daß die Verteilungsfunktion einer Zufallsvariablen im Rahmen einer Stichprobe sich der Verteilungsfunktion der Grundgesamtheit nähert, angenommen oder verworfen. $\mathrm{Da}$ die zu testende Verteilungsfunktion Parameter nicht genau bekannter Werte enthält ( $\mu$-und 
Tab. 2

Erythrocytenporphyrinwerte nach Extraktion der freien Säuren

\begin{tabular}{llll}
\hline Autoren & $\mu \mathrm{g}$ Erythrocytenporphyrine & $\begin{array}{c}\text { Anzahl der } \\
\text { Untersuchungen }\end{array}$ \\
\hline
\end{tabular}

V. D. BERGH und GRotepass (1933) LAGEDER (1936)

SEGGEL (1940)

CARTWRIGHT und Mitarbeiter (1948)

WATSON und Mitarbeiter (1944)

BÉNARD und Mitarbeiter (1949)

WARD und MASON (1950)

VENTURA und MEDURI (1954)

KOSKELO (1959)

\section{WATSON (1950)}

JEFFREY und WATSON (1954)

MEDURI und Mitarbeiter (1958)

KRAMER und Mitarbeiter (1954)

WRANNE (1960)

SCHWARTZ und Mitarbeiter (1960)

RIMINGTON und Mitarbeiter (1963)

JARLIKOWA und Mitarbeiter (1964)

HEILMEYER und Mitarbeiter (1964)

GUTNIAK und Mitarbeiter (1964)

GAJDOS und Mitarbeiter (1965)
LYNCH und MIEDLER (1965)

\section{GESAMTPORPHYRINE}

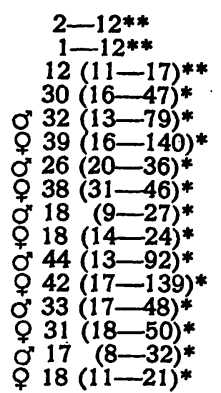

PROTOPORPHYRIN

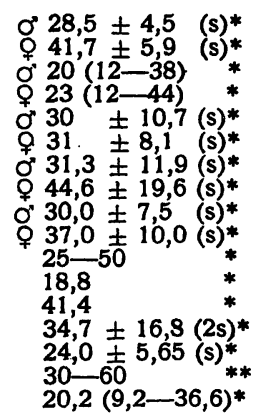

KOPROPORPHYRIN

$\begin{array}{lll}0 & 0,3 \pm 0,39 \text { (s)* } \\ 0 & 0,7 \pm 0,6 & (\mathrm{~s}) *\end{array}$

Ơ $1,1 \pm 0,7$ (s)*

+ $1,3 \pm 0,6$ (s)

$0,71 \pm 0,23(\mathrm{~s}) *$

$0,71 \pm 0,23$ (s)

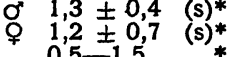

$0,35,1,5 *$

$1,6 \pm 2,4(2 s) *$

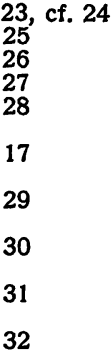

18

33

34

35

12

36

14

16

38

* Bezogen auf $100 \mathrm{ml}$ Erythrocyten („packed cells“)

** Bezogen auf $100 \mathrm{ml}$ Blut.
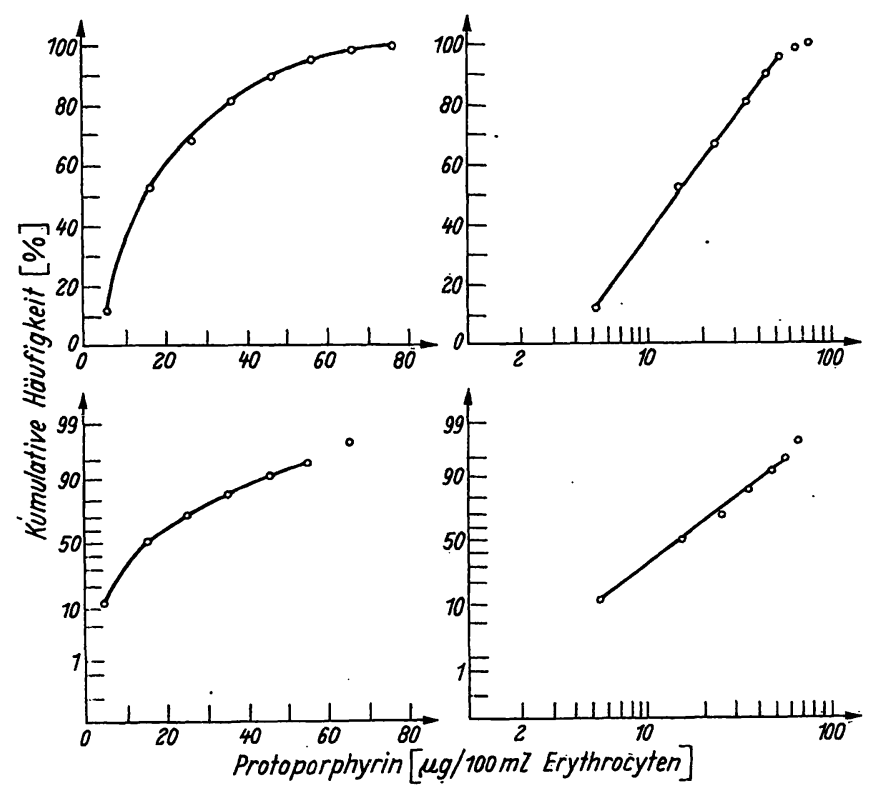

Abb. 1

Normalwerte des Erythrocytenprotoporphyrins nach Analysen àls Methylester. Gegenüberstellung der Resultate auf linearen und logarithmischen Skalen (Abszisse). Oben: Darstellung der prozentualen kumulativen Häufigkeit auf Millimeterpapier. Unten: Darstellung der Häufigkeitssummen in Prozent der G

$\sigma$ ), werden der Stichprobe gemäß der Maximum-LikelihoodSchätzungen $\bar{x}$ und $s$ entnommen (22). Wir verwendeten die Formel:

$$
\chi^{2}=\sum_{i=1}^{k} \frac{\left(n_{1}-n p_{1}\right)^{2}}{n p_{1}}
$$

( $\mathrm{n}=$ Anzahl der Proben; $\mathrm{n}_{\mathrm{L}}=$ Anzahl der Werte pro Klasse; $\mathrm{p}=$ Wahrscheinlichkeit, mit der die Zufallsvariable $\mathrm{X}$ in den

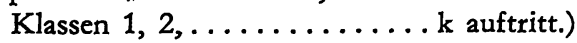

Bei der Verwendung dekadischer Logarithmen der Protoporphyrinwerte $\left(\mu \mathrm{g} / 100 \mathrm{~m} /\right.$ Erythrocyten) ergibt sich ein $\chi^{2}=7,98$.

In der Tabelle der $\chi^{2}$-Verteilung findet man nach Anzahl der Freiheitsgrade $(\mathrm{f}=\mathrm{k}-\mathrm{t}-1 ; \mathrm{t}=$ geschätzte Parameter $\mu \approx \overline{\mathrm{x}}$ und $6 \approx \mathrm{s}$ ) und der Irrtumswahrscheinlichkeit $\alpha$ (Signifikanzzahl) $=5 \%(0,05)$ den Wert $c$.

$\mathrm{c}=12,59$.

Soll die Hypothese angenommen werden, muß $\chi^{2} \leqq \mathrm{c}$ sein. Bei der Rechnung mit den Numeri wird $\chi^{2} \gg c$.

Die Verteilungsfunktion des untersuchten Probekollektivs läßt mit einer Irrtumswahrscheinlichkeit von $5 \%$ auf eine log-normale Verteilung des Erythrocytenprotoporphyrins schließen.

Die Analysendaten, nach Altersklassen gruppiert (16 bis 25, 26-.....75; die Altersklassen bis 65 sind mit je 5 oder mehr Analysen vertreten), zeigten im t-Test nach Student zufällige Unterschiede, d. $h$. $p>0,05$. Der Vergleich der Analysen von Männern und Frauen im $t$-Test bestätigte ebenfalls die NullHypothese.

Die Autoren dankẹn den Herren Drs. J. Kaffarnik (Medizinische Poliklinik der Universität Marburg (Lahn)), E. O. RIECKEN, B. MrLLER (Medizinische Klinik der Universität Marburg (Lahn)) und K. MüLler (Chirurgische Klinik und Poliklinik der Universität Marburg (Lahn)) für die Bereitstellung von Blutproben. 


\title{
Literatur
}

1. Doss, M., J. Chromatog. 30, 265 (1967). - 2. Doss, M. und W. MANNheim, diese Z. 5, 260 (1967). - 3. Goldrick, B. und J. HrRsCH, J. Lipid Res. 4, 482 (1963). - 4. FaLK, J. E., Porphyrins and Metalloporphyrins, Elsevier, Amsterdam-LondonNew York (1964). - 5. BuRT, N. S. und R. J. Rossrter, Biochem. J. 46, 596 (1950). - 6. Doss, M. und U. Bode, J. Chromatog., 35, 248 (1968). - 7. Doss, M. und K. OETrE, diese Z. 3, 125 (1965). 8. Oetre, K. und M. Doss, J. Chromatog. 32, 439 (1968). 9. Ways, P. und D. J. Hanahan, J. Lipid Res. 5, 324 (1964). 10. Doss, M. und H. Bürger, Hoppe-Seyler's Z. physiol. Chem. 348, 936 (1967). - 11. KLENK, E. und M. Doss, Hoppe-Seyler's Z. physiol. Chem. 342, 187 (1965). - 12. WranNe, L., Acta paediatr. 49, Suppl. 124 (1960). - 13. Grinstern, M. und M. M. Wintrobe, J. biol. Chemistry 172, 459 (1948). - 14. Rimington, C., P. N. Morgan, K. Nicholls, J. D. Everall und R. R. Davis, Lancet 1963/II, 318. - 15. Schwartz, S. und H. M. Wikoff. J. biol. Chemistry 194, 563 (1952). - 16. Herlmeyer, L., R. Clotten und L. Heirneyer jr., Die Störungen der Bluthämsynthese mit besonderer Berücksichtigung der sideroachrestischen Anämien und erythropoetischen Porphyrien, Georg Thieme, Stuttgart (1964). - 17. WAtson, C. J., M. Grinstern und V. Hawkinson, J. clin. Invest. 23, 69 (1944):- 18. Watson, C. J., Arch.Int. Med. Chicago 86, 797 (1950). - 19.Doss, M., Dtsch. med. Wschr., im Druck. - 20. Richterich, R., Klinische Chemie, Akademische Verlagsgesellschaft Frankfurt a. M. (1965). - 21. ADAM, J., Einführung in die medizinische Statistik. S. 213. VEB Verlag Volk und Gesundheit, Berlin (1966). - 22. KREYSzIG, E.,
Statistische Methoden und ihre Anwendung, S. 228. Vandenhoeck \& Ruprecht, Göttingen (1965). - 23. VAN DEN BERGH, A. A. H. und W. Grotepass, Klin. Wschr. 12, 586 (1933). 24. Brugsch, J., Porphyrine, S. 171. Johann Ambrosius Barth, Leipzig (1959). - 25. LAGEDER, K., Klin. Wschr. 15, 296 (1936). 26. Sснuмm, C., Zschr. exper. Med. 106, 252 (1939). - 27. SegGer, K. A., Erg. inn. Med. 58, 582 (1940). - 28. Cartwright, G. E., C. M. Huguley, H. Ashenbrucker, J. Fay und M. M. Wintrobe, Blood 3, 501 (1948). - 29. BÉnard, H., A. Gajdos und J. Caniver, Sang. Paris 20, 289 (1949). - 30. WARD, E. und H. L. Mason, J. clin. Invest. 29, 905 (1950). - 31. Ventura, S. und D. Meduri, Haematologica Pavia 38, 747 (1954a). - 32. Koskelo, P., Ann. med. int. Fenniae 48, 55 (1959). - 33. JefFrex, N. R. und D. Watson, Acta haemat. 12, 169 (1954). - 34. MEDURr, D., A. Notario und G. Casirola, Haematologica Pavia 43, 1213 (1958). - 35. Krammer, A., C. E. Cartwright und M. M. Wintrobe, Blood 9, 183 (1954). - 36. Schwartz, S., M. H. Berg, J. Bossenmaier und H. Dinsmore, Determination of porphyrins in biological material. In: Methods of Biochemical Analysis, S. 221. Vol. VIII. ed. by D. Glick, Intersience, New York (1960). - 37. Jarlikowa, E. I., R. I. Jewstignjejewa und W. N. Lusgina, Laborat. Djelo 11, 169 (1954). - 38. Gutniak, O., H. Kozidowa und E. Kowalskr, Lancet 1964/I, 1137. - 39. Gajdos, A., M. Gajdos-Torök, J. M. Mantz und H. Schiradin, Presse méd. Paris 73, 119 (1965). - 40. Lrnch, P. J. und L. J. Mirdler, Arch. Dermat. Syph. 89, 104 (1964).

Dr. M. Doss 355 Marburg (Lahn) Pilgrimstein 2

\section{Isoenzymfraktionen der Fructose-Phosphat-Aldolase ${ }^{1}$ im Serum und verschiedenen Organen des Menschen}

\author{
Isoenzyme der Fructose-Phosphat-Aldolase, I. Mitteilung \\ Von A. L. Dikow \\ Aus der Biochemiscben Abteilung des Wissenschaftlichen Forscbungsinstituts für Onkologie \\ (Direktor: Prof. Dr. N. Antschew) Sofia, Bulgarien
}

(Eingegangen am 1. Dezember 1967)

\begin{abstract}
In der vorliegenden Mitteilung wird ein Verfahren beschirieben, mit dem die Isoenzyme der Fructose-Phosphat-Aldolasen ${ }^{1}$ ) in Serum und Organen des Menschen durch Agarosegelelektrophorese und darauffolgende Inkubation mit Substratlösung getrennt und spezifisch nachgewiesen werden können. Nach der Elektrophorese wird das Gel bei $37^{\circ}$ in einer Lösung von FDP, Natrium arsenat, NAD, GAP-DH, Diaphorase und NBT inkubiert. Es wurden vier Isoenzymfraktionen der ALD im Serum gesunder Probanden gefunden, und źwar von der Anode zur Kathode hin als I, II, III und IV bezeichnet. Sie befinden sich dementsprechend in den Zonen der Albumine, $\alpha_{2}-, \beta_{2}-$ und $\gamma$-Globuline des Serums. Nach Berechnung der quantitativen Werte der einzelnen Isoenzymfraktionen wurden die Ergebnisse einer statistischen Bearbeitung unterworfen und Mittelwert, Standardabweichung und mit der Methode der Perzentile auch die Normalwerte der Isoenzyme der ALD im Serum berechnet. Bei verschiedenen Krankheiten steigt ihre Zahl auf 8 . In normalen Organen wurden 10 Isoenzymfraktionen beobachtet.
\end{abstract}

1) Abkürzungen und Enzy'me: Aldolasen: ALD = Aldolase, MALD $=$ Muskelaldolase, LALD $=$ Leberaldolase, Aldolase " $\mathrm{A}^{\text {“ }}$ Fructose-1,6-diphosphat D-Glycerinaldehyd-3-phosphat Lyase (EC 4.1.2.13), Aldolase „B" - Ketose-1-phosphat Aldehyd Lyase (EC 4.1.2.7), GAP-DH = Glycerinaldehyd-3-phosphat-Dehydrogenase = D-Glycerinaldehyd-3-phosphat: NAD Oxydoreduktase (phos- phorylierend) (EC 1.2.1.12), Diaphorase (NAD- $\mathrm{H}_{2}$ : Lipoamid Oxydoreduktase EC 1.6.4.3), FDP - Fructose-1,6-diphosphat, FMP - Fructose-1-phosphat, GAP = Glycerinaldehyd-3-phosphat, DAP = Dihydroxyacetonphosphat, NBT '- NitroblauTetrazolium. 